\title{
Astrophysical parameters of 14 open clusters projected close to the Galactic plane
}

\author{
D. Camargo, C. Bonatto, and E. Bica
}

\begin{abstract}
Universidade Federal do Rio Grande do Sul, Departamento de Astronomia, CP 15051, RS, Porto Alegre 91501-970, Brazil e-mail: denilso.camargo@ufrgs.br; [bica;charles]@if.ufrgs.br
\end{abstract}

Received 29 June 2009 / Accepted 9 September 2009

\begin{abstract}
Aims. Astrophysical parameters (age, reddening, distance, core and cluster radii) of 14 open clusters (OCs) projected close to the Galactic plane are derived with 2MASS photometry. The OCs are Be 63, Be 84, Cz 6, Cz 7, Cz 12, Ru 141, Ru 144, Ru 172, FSR 101, FSR 1430, FSR 1471, FSR 162, FSR 178, and FSR 198. The OCs Be 63, Be 84, Ru 141, Ru 144, and Ru 172 are studied in more detail than in previous works, while the others have astrophysical parameters derived for the first time.

Methods. We analyse the colour-magnitude diagrams (CMDs) and stellar radial density profiles (RDPs) built after field-star decontamination and colour-magnitude filtered photometry. Field-star decontamination is applied to uncover the cluster's intrinsic CMD morphology, and colour-magnitude filters are used to isolate stars with a high probability of being cluster members in view of structural analyses.

Results. The open clusters of the sample are located at $d_{\odot}=1.6-7.1 \mathrm{kpc}$ from the Sun and at Galactocentric distances 5.5-11.8 kpc, with age in the range $10 \mathrm{Myr}$ to $1.5 \mathrm{Gyr}$ and reddening $E(B-V)$ in the range $0.19-2.56 \mathrm{mag}$. The core and cluster radii are in the range $0.27-1.88 \mathrm{pc}$ and $2.2-11.27 \mathrm{pc}$, respectively. Cz 6 and FSR 198 are the youngest OCs of this sample, with a population of pre-main sequence (PMS) stars, while FSR 178 is the oldest cluster.
\end{abstract}

Key words. open clusters and associations: general - Galaxy: stellar content - Galaxy: structure

\section{Introduction}

Open clusters (OCs) are self-gravitating stellar systems formed along the gas- and dust-rich Galactic plane. They contain from tens to a few thousand stars distributed in an approximately spherical structure of up to a few parsecs in radius. The structure of most OCs can be roughly described by two subsystems, the dense core, and the sparse halo (Bonatto \& Bica 2005, and references therein).

Because it is relatively simple to estimate the age and distance of OCs, they have become fundamental probes of Galactic disc properties (Lyngå 1982; Janes \& Phelps 1994; Friel 1995; Bonatto et al. 2006a; Piskunov et al. 2006; Bica et al. 2006b). However, the proximity of most OCs to the plane and the corresponding high values of reddening and field-star contamination usually restrict this analysis to the more populous and/or to those located at most a few kpc from the Sun (Bonatto et al. 2006a).

Detailed analysis of OCs and the derivation of their astrophysical parameters will contribute to future disc studies by unveiling the properties of individual OCs. These parameters, in turn, can help constrain theories of molecular cloud fragmentation, star formation, and dynamical and stellar evolution.

The stellar content of a cluster evolves with time, and internal and external interactions affect the properties of individual clusters. Presently the age distribution of star clusters in the disc of the Galaxy can only be explained if these objects are subjected to disruption timescales of a few times $10^{8} \mathrm{yrs}$ (Oort 1957; Wielen 1971, 1988; Lamers et al. 2004). Open clusters experience external perturbations by giant molecular clouds (GMCs) and by spiral arms and other disc-density perturbations.
To understand how OCs evolve, it is important to take the effect of these external perturbations into account (Gieles et al. 2007).

Cluster disruption is a gradual process with different mechanisms acting simultaneously. Disruption of OCs due to internal processes are characterised by three distinct phases. These phases and their typical timescales are: (i) infant mortality ( $\left.\sim 10^{7} \mathrm{yr}\right)$; (ii) stellar evolution $\left(\sim 10^{8} \mathrm{yr}\right)$ and (iii) tidal relaxation $\left(\sim 10^{9} \mathrm{yr}\right)$. During all three phases, there are additional external tidal perturbations from e.g. GMCs and disc-shocking that heat the cluster and speed up the process of disruption. However, these perturbations operate on longer timescales for cluster populations and so are more important for tidal relaxation (Lamers et al. 2004, 2005; Lamers \& Gieles 2006). The combination of these effects results in a time-decreasing cluster mass, until either its complete disruption or a remnant (Pavani \& Bica 2007, and references therein) is left.

Probably reflecting the Galactocentric-dependence of most of the disruptive effects, the Galaxy presents a spatial asymmetry in the age distribution of OCs. Indeed, van den Bergh \& McClure (1980) noted that OCs older than $\gtrsim 1$ Gyr tend to be concentrated in the anti-centre, a region with a low density of GMCs. In this sense, the combined effect of tidal field and encounters with GMCs has been invoked to explain the lack of old OCs in the solar neighbourhood (Gieles et al. 2006, and references therein). Near the solar circle most OCs appear to dissolve on a timescale shorter than $\approx 1$ Gyr (Bergond et al. 2001; Bonatto et al. 2006a). In more central parts, interactions with the disc, the enhanced tidal pull of the Galactic bulge, and the high frequency of collisions with GMCs tend to destroy the poorly populated OCs on a timescale of a few $10^{8} \mathrm{yr}$ (e.g. 

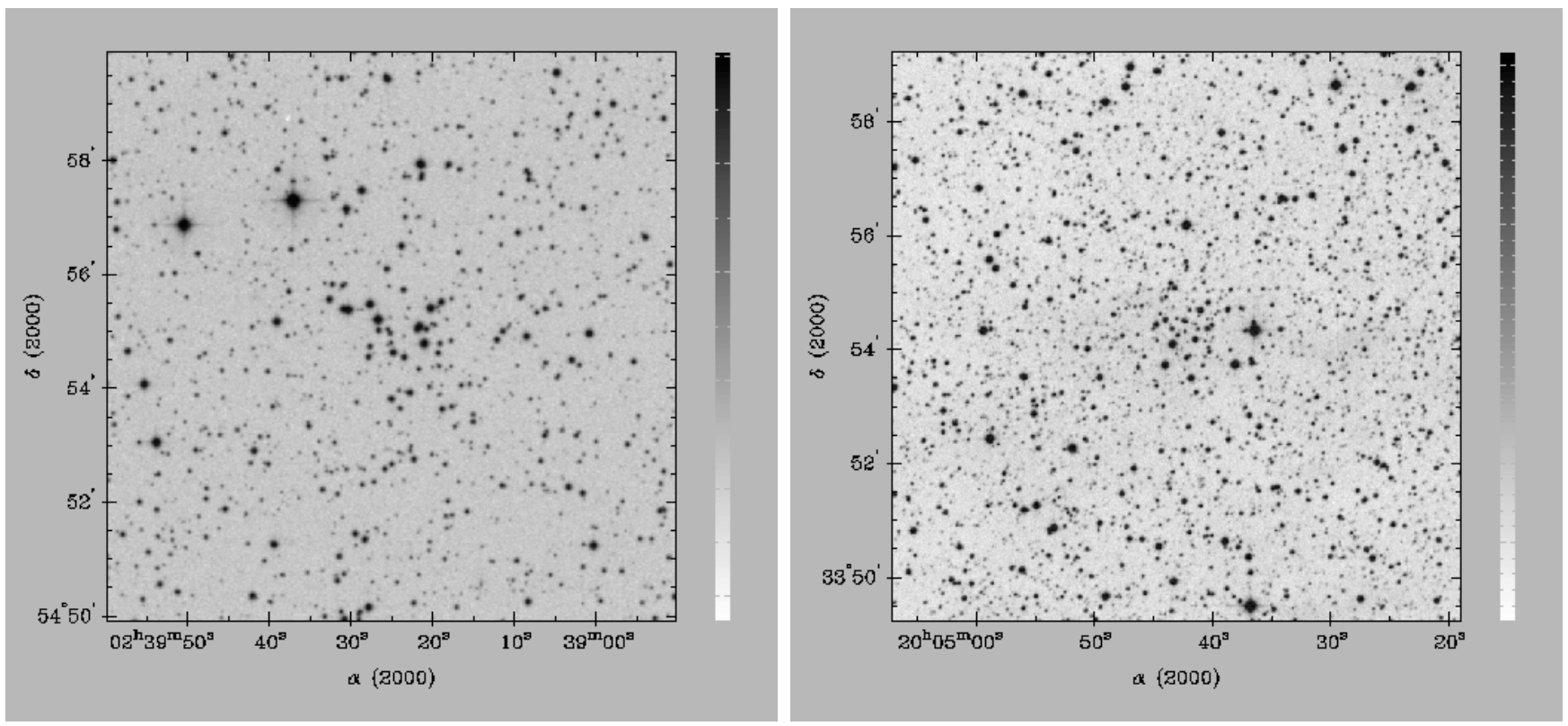

Fig. 1. Left panel: $10^{\prime} \times 10^{\prime}$ XDSS $R$ image of Cz 12. Right panel: $10^{\prime} \times 10^{\prime}$ XDSS $R$ image of Be 84. Images centred on the optimised coordinates.

Bergond et al. 2001). Maciejewski \& Niedzielski (2007) studied a large sample of open clusters, in general not previously studied, to derive fundamental parameters, similar to the present analysis.

This paper is organised as follows. In Sect. 2 we provide general data on the target clusters. In Sect. 3 we obtain the 2MASS photometry, introduce the tools, CMDs and field-star decontamination algorithm, and derive fundamental parameters of the OCs candidates. In Sect. 4 we discuss the stellar radial density profiles (RDPs), colour-magnitude filters, and derive structural parameters. In Sect. 5 we discuss properties of the OCs, and concluding remarks are given in Sect. 6 .

\section{The target open clusters and candidates}

The OCs selected for the present analysis are shown in Table 1. These objects are listed in the OC catalogues WEBDA (Mermilliod 1996) and Froebrich et al. (2007). According to the OC catalogues WEBDA (Mermilliod 1996) and DAML02 (Dias et al. 2002), the target objects do not have published astrophysical parameters, except for Be 63, Be 84, Ru 141, Ru 144, and $\mathrm{Ru}$ 172. The optical sample was chosen as a challenge to our analysis tools (Sect. 3 and references therein). They are low Galactic-latitude clusters that are often heavily contaminated, and poorly populated, and that have differential reddening and a few previous parameter determinations if any at all. The infrared candidates (Froebrich et al. 2007-FSR) were selected from eye inspections that we made on the 2MASS Atlas for promising candidates. In addition, we analysed some of FSR's quality flag Q0-Q3 objects. All FSR objects that we collected in the present study were concluded to be star clusters (Sects. 3 and 4).

Kharchenko et al. (2005) employed the ASCC-2.5 catalogue to derive parameters for 520 OCs, using proper motion and photometric criteria to separate probable members from field stars. However, owing to distance and reddening limitations, the fainter cluster parameters rely on a few stars. For Ru 141 they derived $E(B-V)=0.57, d_{\odot}=5.5 \mathrm{kpc}$, and age $\approx 8 \mathrm{Myr}$. For Ru 172 they derived $E(B-V)=0.20, d_{\odot}=1.1 \mathrm{kpc}$, and age $\approx 0.8 \mathrm{Gyr}$.
Tadross (2008) present astrophysical parameters of 24 open clusters of the Berkeley list, using 2MASS photometry and the proper motions of the Naval Observatory Merged Astrometric Dataset (NOMAD). For Be 63 he derived $E(B-V)=0.90, d_{\odot}=$ $3.3 \mathrm{kpc}, R_{\mathrm{GC}}=11.0 \mathrm{kpc}$, and age $\approx 500 \mathrm{Myr}$. For Be 84 he derived $E(B-V)=0.76, d_{\odot}=2.0 \mathrm{kpc}, R_{\mathrm{GC}}=8.1 \mathrm{kpc}$, and age $\approx 120$ Myr.

In Fig. 1, we illustrate cluster $\mathrm{XDSS}^{1}$ images in the $R$ band of $\mathrm{Cz} 12$ and Be 84. In Fig. 2, we show 2MASS images in the $K$ band of the IR clusters FSR 198 and FSR 1430. Be 84, FSR 198, and Ru 144 present significant differential reddening, consistent with their low Galactic latitude (Table 1). Ru 172 appears to present a similar effect, but only in the background, and the FSR 1430 image shows a reddening gradient in the north/south direction. Ru 144 and Ru 141 show absorption effects in the background/foreground. Ru 172 seems to be offcentred in the optical image, probably owing to absorption.

\section{The 2MASS photometry}

The 2MASS ${ }^{2}$ catalogue (Skrutskie et al. 2006) was employed in the present work because of the homogeneity and the possibility of large-area data extractions. Also, part of the sample cannot be studied in the optical. VizieR ${ }^{3}$ was used to extract $J, H$, and $K_{\mathrm{s}}$ 2MASS photometry. Our previous experience shows that, as long as no other cluster is present in the field and differential absorption is not prohibitive, such large extraction areas provide the required statistics for field-star characterisation. To maximise the statistical significance and representativeness of background star counts, we use a wide external ring to represent the stellar comparison field. The RDPs produced with the WEBDA coordinates presented in general a dip in the innermost bin. For these we searched for new coordinates that maximise the star-counts

\footnotetext{
1 Extracted from the Canadian Astronomy Data Centre (CADC), at http: //cadcwww. dao.nrc.ca/

2 The Two Micron All Sky Survey, available at wwW . . ipac . caltech . edu/2mass/releases/allsky/ 3 http://vizier.u-strasbg.fr/viz-bin/VizieR? - source=II/246
} 

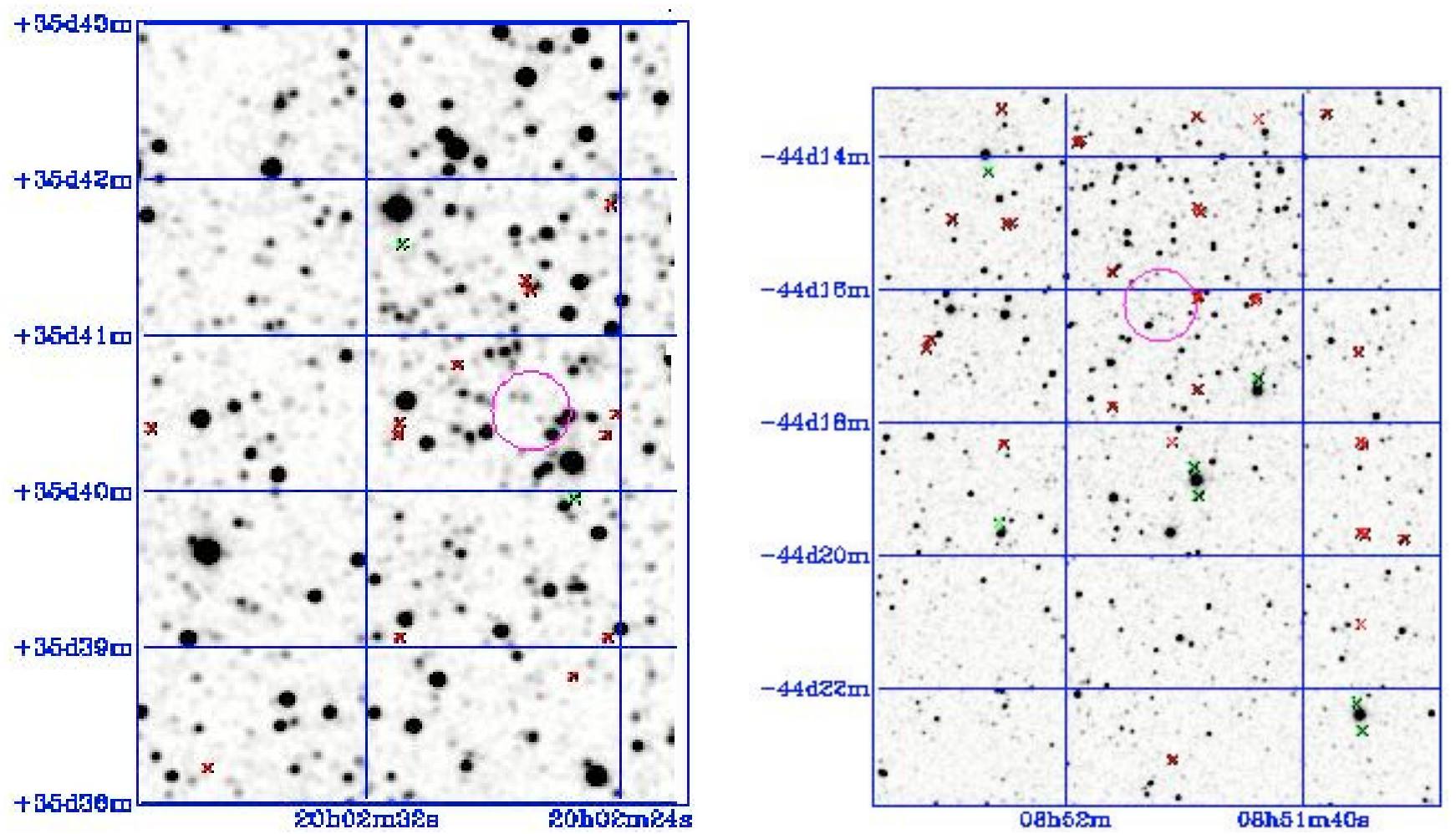

Fig. 2. Left panel: 2 MASS $K_{\mathrm{S}}$ image $5^{\prime} \times 5^{\prime}$ of FSR 198. Right panel: 2 MASS $K_{\mathrm{S}}$ image $15^{\prime} \times 15^{\prime}$ of FSR 1430 . Images centred on the optimised coordinates. The small circle indicates the cluster central region.

Table 1. Literature and presently optimised coordinates.

\begin{tabular}{|c|c|c|c|c|c|c|c|c|}
\hline \multirow[b]{2}{*}{ Cluster } & \multicolumn{4}{|c|}{ Literature } & \multicolumn{4}{|c|}{ This paper } \\
\hline & $\begin{array}{r}\alpha(2000) \\
(\mathrm{h} \mathrm{m} \mathrm{s})\end{array}$ & $\begin{array}{r}\delta(2000) \\
\left({ }^{\circ}{ }^{\prime \prime \prime}\right)\end{array}$ & $\begin{array}{r}\ell \\
\left(^{\circ}\right)\end{array}$ & $\begin{array}{r}b \\
\left(^{\circ}\right)\end{array}$ & $\begin{array}{r}\alpha(2000) \\
(\mathrm{h} \mathrm{m} \mathrm{s})\end{array}$ & $\begin{array}{r}\delta(2000) \\
\left({ }^{\prime}, \prime \prime\right)\end{array}$ & $\begin{array}{r}\ell \\
\left({ }^{\circ}\right)\end{array}$ & $\begin{array}{r}b \\
\left({ }^{\circ}\right)\end{array}$ \\
\hline Be 63 & 021936 & 634300 & 132.506 & 2.49 & 021930.8 & 634343 & 132.49 & 2.50 \\
\hline Be 84 & 200443 & 335418 & 70.924 & 1.27 & 200443 & 335415 & 70.92 & 1.27 \\
\hline $\mathrm{Cz} 6$ & 020200 & 625000 & 130.887 & 1.05 & 020157 & 625048 & 130.87 & 1.07 \\
\hline $\mathrm{Cz} 7$ & 020224 & 621500 & 131.159 & 0.52 & 020301 & 621520 & 131.16 & 0.53 \\
\hline $\mathrm{Cz} 12$ & 023912 & 545500 & 138.079 & -4.75 & 023925 & 545455 & 138.11 & -4.74 \\
\hline Ru 141 & 183119 & -121911 & 19.69 & -1.20 & 183123 & -121750 & 19.72 & -1.21 \\
\hline Ru 144 & 183334 & -112500 & 20.749 & -1.27 & 183333 & -112509 & 20.74 & -1.27 \\
\hline Ru 172 & 201134 & 353559 & 73.11 & 1.01 & 201139 & 353730 & 73.14 & 1.00 \\
\hline FSR 101 & 184914 & 024606 & 35.147 & 1.74 & 184914 & 024606 & 35.14 & 1.74 \\
\hline FSR 1430 & 085152 & -441556 & 264.65 & 0.08 & 085152 & -441614 & 264.66 & 0.07 \\
\hline FSR 1471 & 092408 & -472039 & 270.72 & 2.14 & 092404 & -472056 & 270.71 & 2.13 \\
\hline FSR 162 & 200132 & 251406 & 63.211 & -2.75 & 200126 & 251230 & 63.17 & -2.74 \\
\hline FSR 178 & 201307 & 290712 & 67.877 & -2.83 & 20138.2 & 290724 & 67.88 & -2.83 \\
\hline FSR 198 & 200224 & 354119 & 72.184 & 2.62 & 200227 & 354031 & 72.17 & 2.60 \\
\hline
\end{tabular}

at the centre. For each cluster we made circular extractions centred on the optimised coordinates of the clusters. The WEBDA and optimised central coordinates are given in Table 1.

The statistical significance of astrophysical parameters depends directly on the quality and depth of the photometry (Bonatto et al. 2004b, 2005). As a photometric quality constraint, 2MASS extractions were restricted to stars with magnitudes (i) brighter than those of the $99.9 \%$ Point Source Catalogue completeness limit in the cluster direction; and (ii) with errors in $J, H$, and $K_{\mathrm{s}}$ smaller than $0.1 \mathrm{mag}$. The $99.9 \%$ completeness limits are different for each cluster, varying with Galactic coordinates. A typical distribution of uncertainties as a function of magnitude, for objects projected towards the central parts of the Galaxy, can be found in Bonatto \& Bica (2007a). About 75-85\% of the stars have errors below 0.06 mag.

\subsection{Field-star decontamination}

The CMD is an important tool for searching for the fundamental parameters of the star clusters, but the field-star contamination is an important source of uncertainty, particularly for low-latitude OCs and/or those projected against the bulge. Our sample of OCs is located in crowded disc zones near the plane, and because of the low latitude, field stars contaminate the CMDs, especially at faint magnitudes and red colours.

To uncover the intrinsic cluster CMD morphology, we use the field-star decontamination procedure described in Bonatto \& Bica (2007b), previously applied in the analysis of low-contrast (Bica \& Bonatto 2005), embedded (Bonatto et al. 2006a), young (Bonatto et al. 2006c), faint (Bica et al. 2006b), old (Bonatto \& Bica 2007a), or in dense-fields (Bonatto \& Bica 2007b) OCs. 
Table 2. Previous determinations.

\begin{tabular}{lrrrr}
\hline \hline Cluster & $\begin{array}{r}\text { Age } \\
(\mathrm{Myr})\end{array}$ & $\begin{array}{r}E(B-V) \\
(\mathrm{mag}) \\
(2)\end{array}$ & $\begin{array}{r}d_{\odot} \\
(\mathrm{kpc})\end{array}$ & Source \\
$(1)$ & 500 & 0.90 & 3.3 & $(5)$ \\
\hline Be 63 & 500 & $(1)$ \\
Be 84 & 120 & 0.76 & 2.0 & $(1)$ \\
Ru 141 & 8 & 0.57 & 5.5 & $(2)$ \\
Ru 144 & 151 & 0.32 & - & - \\
Ru 172 & 800 & 0.20 & 1.1 & $(2)$ \\
\hline
\end{tabular}

Notes. References: (1) - Tadross (2008); (2) - Kharchenko et al. (2005).

The algorithm works on a statistical basis that takes the relative number densities of stars in a cluster region and offset field into account. The algorithm works with three dimensions, the $J$ magnitude and the $(J-H)$ and $\left(J-K_{\mathrm{s}}\right)$ colours, considering as well the respective $1 \sigma$ uncertainties in the 2MASS bands. These colours provide the maximum discrimination among CMD sequences for star clusters of different ages (e.g. Bonatto et al. 2004a).

Basically, the algorithm (i) divides the full range of magnitude and colours of a given CMD into a 3D grid whose cubic cells have axes along the $J,(J-H)$, and $\left(J-K_{\mathrm{s}}\right)$ directions; (ii) computes the expected number density of field stars in each cell based on the number of comparison field stars (within $1 \sigma$ Poisson fluctuation) with magnitude and colours compatible with those of the cell; and (iii) subtracts the expected number of field stars from each cell. Consequently, this method is sensitive to local variations in field star contamination with magnitude and colours. Cell dimensions are $\Delta J=1.0$, and $\Delta(J-H)=\Delta\left(J-K_{\mathrm{s}}\right)=0.15$, which are adequate to allow sufficient star-count statistics in individual cells and preserve the morphology of the CMD evolutionary sequences. The dimensions of the colour/magnitude cells can be changed subsequently so that the total number of stars subtracted throughout the whole cluster area matches the expected one, within the $1 \sigma$ Poisson fluctuation.

Three different grid specifications in each dimension are used to minimise potential artifacts introduced by the choice of parameters, thus resulting in 27 different outputs. They occur because for a CMD grid beginning at magnitude $J_{0}$ (with cell width $\Delta J$ ), we also include additional runs for cell centres shifted by $J_{0} \pm \frac{1}{3} \Delta J$. Also when considering the same strategy applied to the 2 colours, we end up with 27 outputs. The average number of probable cluster stars $\left\langle N_{\mathrm{cl}}\right\rangle$ is computed from these outputs. Typical standard deviations of $\left\langle N_{\mathrm{cl}}\right\rangle$ are at the $\approx 2.5 \%$ level. The final field-star decontaminated CMD contains the $\left\langle N_{\mathrm{cl}}\right\rangle$ stars with the highest number frequencies. Stars that remain in the CMD after the field star decontamination are in cells where the stellar density presents a clear excess over the field. Consequently, they have a significant probability of being cluster members. Further details on the algorithm, including discussions of subtraction efficiency and limitations, are given in Bonatto \& Bica (2007b).

Bica et al. (2008) introduce the parameter $N_{1 \sigma}$ which corresponds to the ratio of the number of stars in the decontaminated CMD with respect to the $1 \sigma$ Poisson fluctuation measured in the observed CMD. By definition, CMDs of overdensities must have $N_{1 \sigma}>1$. It is expected that CMDs of star clusters have $N_{1 \sigma}$ significantly larger than 1 . The $N_{1 \sigma}$ values for the present sample are given in Col. 3 of Table 2.
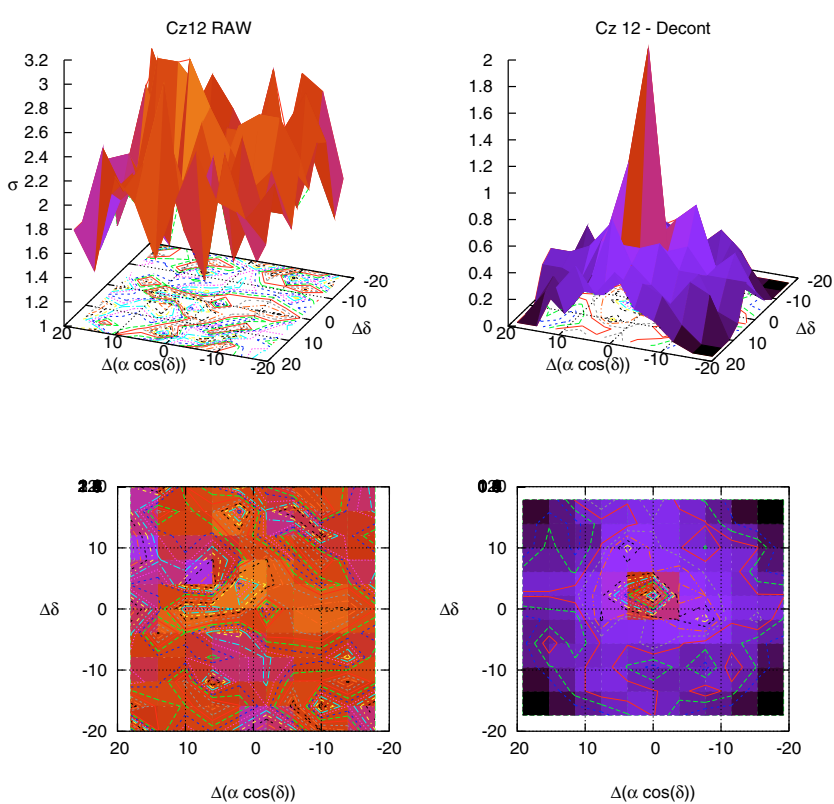

Fig. 3. Top panels: stellar surface-density $\sigma\left(\operatorname{stars} \operatorname{arcmin}^{-2}\right)$ of $\mathrm{Cz} 12$, computed for a mesh size of $3^{\prime} \times 3^{\prime}$, centred on the coordinates in Table 1. Bottom: the corresponding isopleth surfaces. Left: observed (raw) photometry. Right: decontaminated photometry.
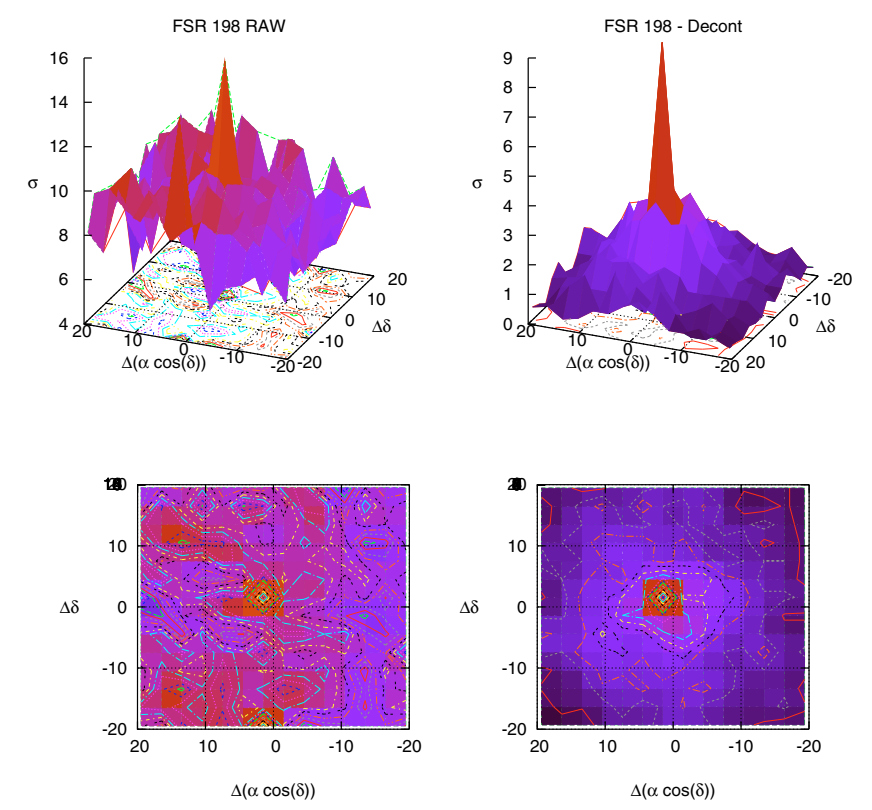

Fig. 4. Same as Fig. 3 for FSR 198.

\subsection{Fundamental parameters}

Astrophysical fundamental parameters are derived with solarmetallicity Padova isochrones (Girardi et al. 2002) computed with the 2MASS $J, H$, and $K_{\mathrm{s}}$ filters. The 2MASS transmission filters produced isochrones very similar to the Johnson-KronCousins ones (e.g. Bessel \& Brett 1988), with differences of at most 0.01 in $(J-H)$ (Bonatto et al. 2004a). The best fits are superimposed on decontaminated CMDs. Parameters derived from the isochrone fit are the observed distance modulus $(m-M)_{J}$ and reddening $E(J-H)$, which converts to $E(B-V)$ and $A_{V}$ with the relations $A_{J} / A_{V}=0.276, A_{H} / A_{V}=0.176, A_{K_{\mathrm{s}}} / A_{V}=0.118$, $A_{J}=2.76 \times E(J-H)$, and $E(J-H)=0.33 \times E(B-V)$ (Dutra et al. 2002), assuming a constant total-to-selective absorption 


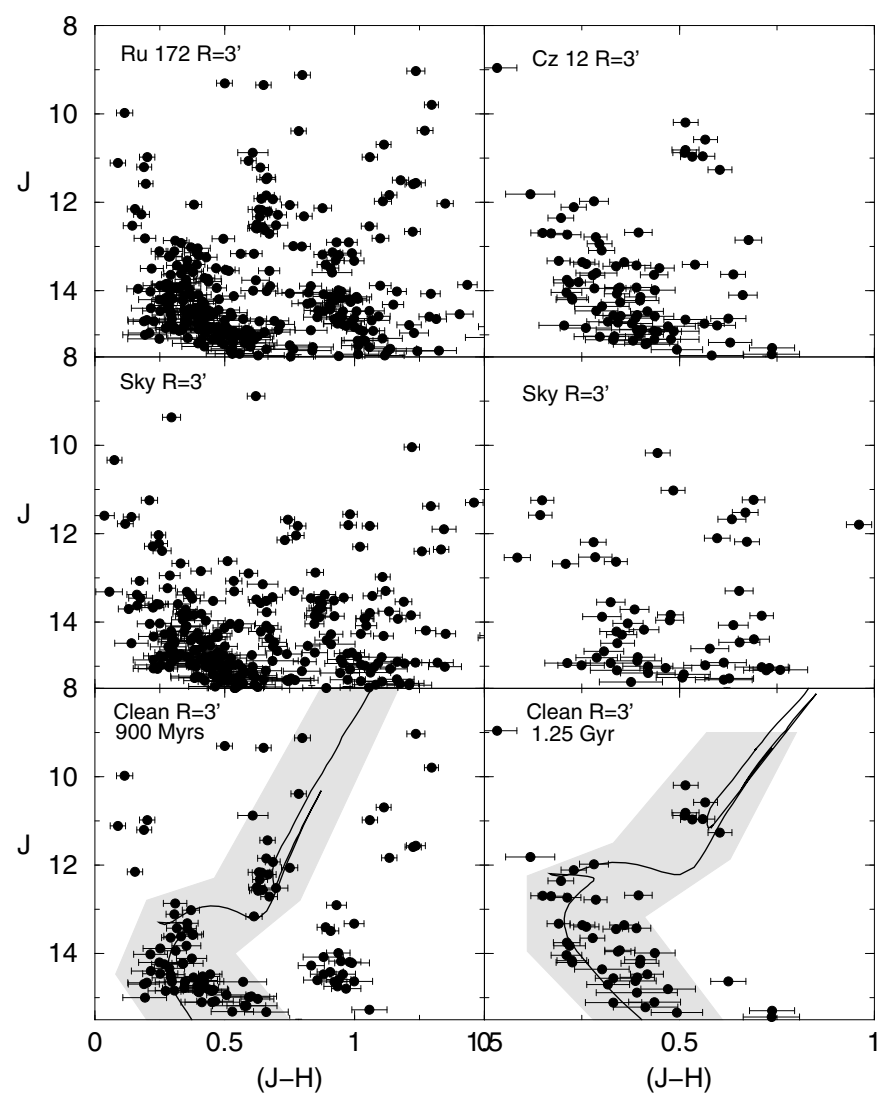

Fig. 5. 2MASS CMDs extracted from the $R=3^{\prime}$ region of $\mathrm{Ru} 172$ and $\mathrm{Cz} 12$, respectively. Top panels: observed CMDs $J \times(J-H)$. Middle panels: equal area comparison field. Bottom panels: field-star decontaminated CMDs fitted with the 900 Myr Padova isochrone (solid line) for $\mathrm{Ru} 172$ and $1.25 \mathrm{Gyr}$ for $\mathrm{Cz} 12$. The colour-magnitude filter used to isolate cluster MS/evolved stars is shown as a shaded region.

ratio $R_{V}=3.1$. The resulting age, $E(B-V), d_{\odot}$, and $R_{\mathrm{GC}}$ are given in Cols. 3 to 6 of Table 2. FSR 198 and Cz 6 presents a significant population of pre-main sequence (PMS) stars. Isochrones of Siess et al. (2000) are used to characterise the PMS sequences of these objects (Figs. 7 and 8).

In Fig. 5 we present the $J \times(J-H)$ CMDs extracted from a region $R=3^{\prime}$ centred on the optimised coordinates of Ru 172 and $\mathrm{Cz} 12$ (top-panel). In the middle panels we show the background field corresponding to a ring with the same area as the central region. In the bottom panels we built the field-star decontaminated CMDs.

Both $\mathrm{Ru} 172$ and $\mathrm{Cz} 12$ can be recognised as a cluster by the presence of the MS and a prominent giant clump. These features are not present in the comparison field (Fig. 5 middle panels). Figure 6 shows 9 OCs. FSR 162 and FSR 178 are probable remnant OCs. The present sample of OCs has in general low stellar-density contrast with respect to the background owing to the projection close to the plane (Table 1).

$\mathrm{Cz} 7$ is not a populous cluster, but the results point to a relatively young OC (age $\approx 220 \mathrm{Myr}$ ). The decontamination leads to an age of $\approx 30$ Myr for Be 63, but deep observations are required for more conclusive results. Deeper photometry is essential in most cases, especially for faint and/or distant OCs, close to the plane, affected by 2 MASS completeness limits. $\mathrm{Cz} 6$ and FSR 198 are very young OCs (10 Myr). Be 84 turns out to be moderately young (age $\approx 360 \mathrm{Myr}$ ), similar to $\mathrm{Ru} 144$ with 450 Myr. After decontamination, Ru 141 corresponds to

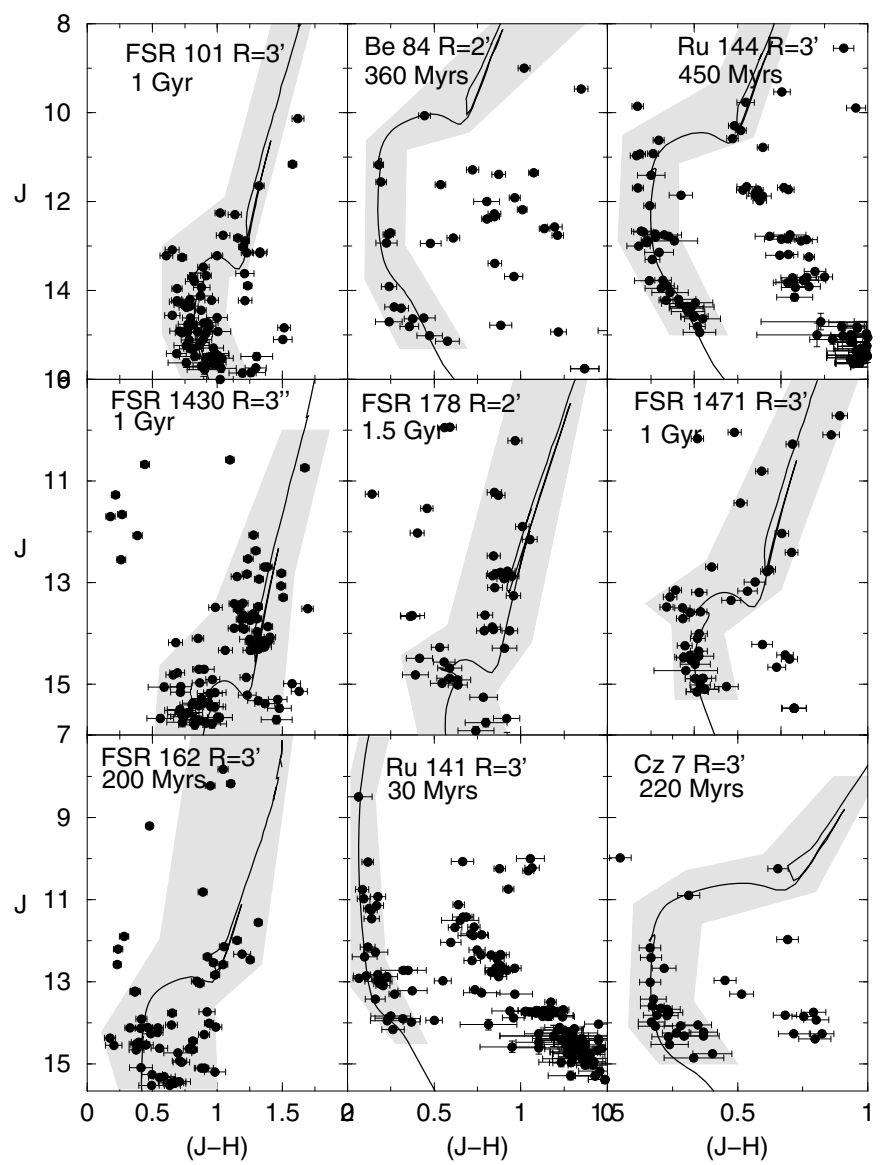

Fig. 6. Same as Fig. 5 for the decontaminated $J \times(J-H)$ CMDs of the central regions of each object.

the bluest sequence in that diagram (age $\approx 30 \mathrm{Myr}$ ). Since $\mathrm{Ru}$ 141, Ru 144, and Ru 172 are located at very low Galactic latitudes (Table 1), important absorption variations across the cluster area and/or background may occur, which can produce residual effects in decontaminated CMDs. In particular, Ru 141 has a strong absorption in $J$ for the background stars at $\approx 2.5^{\prime}$ to the northeast. It also has a strong absorption in $B$ at $\approx 6^{\prime}$ to the east. Indeed, $\mathrm{Ru} 141$ shows significant residuals in the CMD (Fig. 6). We point out that the field of Ru 141 also contains the OC Ru 142 at $\approx 10^{\prime}$. Finally, FSR 162 is a faint and distant OC $\left(d_{\odot}=7.1 \mathrm{kpc}\right)$. Only field decontamination made possible to probe cluster properties. Their OC nature is further supported by their decontaminated structural properties (Sect. 4).

We note that there are some differences in the fundamental parameters with respect to previous works (e.g. Kharchenko et al. 2005; Tadross et al. 2002), especially for cluster age. This occurs especially for young clusters in which field contamination has not been properly taken into account. In these cases, PMS stars in conjunction with important field contamination may mimic older ages. A clear example is FSR 198 (Fig. 7).

\section{Structural parameters}

Structural parameters have been derived by means of the stellar RDPs, defined as the projected number of stars per area around the cluster centre. RDPs are built with stars selected after applying the respective CM filter to the observed photometry. The colour-magnitude filters (CM filters) are shown in Figs. 5-9 

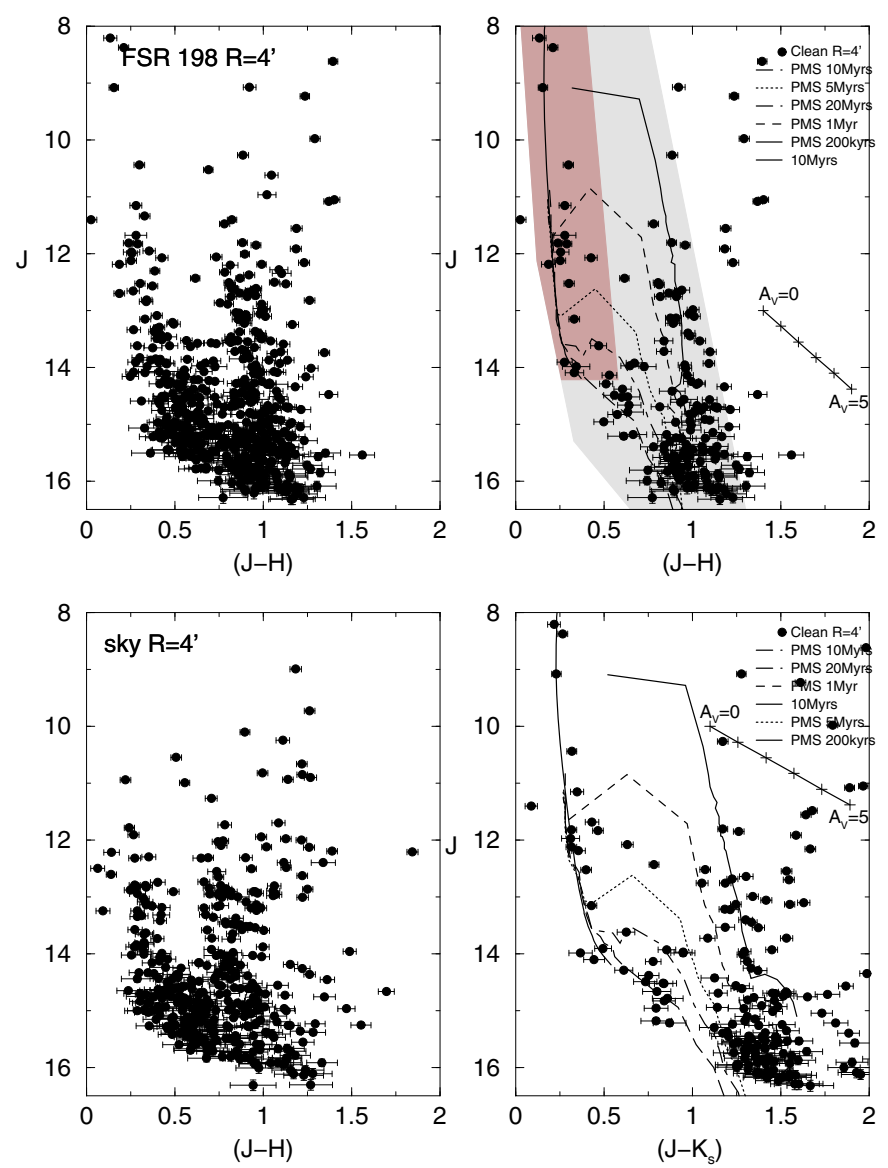

Fig. 7. 2MASS CMDs extracted from the $R=4^{\prime}$ region of FSR 198 . Top panels: observed CMDs $J \times(J-H)$ (left) and field star decontaminated CMDs fitted with MS + PMS isochrone solutions. Shaded polygons show the MS (dark) and PMS (light) colour-magnitude filter used to isolate cluster MS/evolved stars (right). Bottom panels: equal area comparison field (left) and $J \times\left(J-K_{s}\right)$ field star decontaminated CMDs fitted with MS + PMS isochrone solutions.Reddening vectors for $A_{V}=0-5$ are shown in decontaminated CMDs.

Table 3. Derived fundamental parameters.

\begin{tabular}{lrrrrr}
\hline \hline Cluster & $\begin{array}{r}\text { Age } \\
(\mathrm{Gyr})\end{array}$ & $N_{1 \sigma}$ & $\begin{array}{r}E(B-V) \\
(\mathrm{mag})\end{array}$ & $\begin{array}{r}d_{\odot} \\
(\mathrm{kpc})\end{array}$ & $\begin{array}{r}R_{\mathrm{GC}} \\
(\mathrm{kpc})\end{array}$ \\
$(1)$ & $(2)$ & $(3)$ & $(4)$ & $(6)$ \\
\hline Be 63 & $0.03 \pm 0.01$ & 3.7 & $0.96 \pm 0.03$ & 5.7 & 11.8 \\
Be 84 & $0.36 \pm 0.05$ & 5.4 & $0.58 \pm 0.06$ & 1.7 & 6.8 \\
Cz 6 & $0.01 \pm 0.005$ & 8.3 & $0.26 \pm 0.03$ & 2.7 & 6.9 \\
Cz 7 & $0.22 \pm 0.05$ & 3.6 & $0.70 \pm 0.03$ & 3.3 & 9.7 \\
Cz 12 & $1.25 \pm 0.4$ & 4.6 & $0.26 \pm 0.03$ & 2.0 & 8.8 \\
Ru 141 & $0.03 \pm 0.02$ & 12.8 & $0.45 \pm 0.1$ & 1.8 & 5.5 \\
Ru 144 & $0.45 \pm 0.1$ & 7.7 & $0.77 \pm 0.1$ & 1.6 & 5.7 \\
Ru 172 & $0.9 \pm 0.2$ & 5.6 & $0.64 \pm 0.06$ & 3.1 & 7.0 \\
FSR 101 & $0.9 \pm 0.2$ & 7.1 & $2.37 \pm 0.03$ & 1.9 & 7.1 \\
FSR 1430 & $1.0 \pm 0.3$ & 8.3 & $2.56 \pm 0.03$ & 3.6 & 8.4 \\
FSR 1471 & $1.0 \pm 0.2$ & 6.2 & $1.22 \pm 0.02$ & 2.7 & 7.7 \\
FSR 162 & $0.2 \pm 0.05$ & 3.5 & $1.57 \pm 0.03$ & 7.1 & 7.5 \\
FSR 178 & $1.5 \pm 0.5$ & 3.9 & $1.34 \pm 0.03$ & 3.7 & 6.4 \\
FSR 198 & $0.01 \pm 0.005$ & 6.6 & $0.96 \pm 0.03$ & 1.7 & 6.9 \\
\hline
\end{tabular}

Notes. The parameter $N_{1 \sigma}$ corresponds to the ratio of the number of stars in the decontaminated CMD with respect to the $1 \sigma$ Poisson fluctuation measured in the observed CMD (Bica et al. 2008). Column 4: reddening in the cluster's central region. Column $6: R_{\mathrm{GC}}$ calculated using $R_{\odot}=$ $7.2 \mathrm{kpc}$ (Bica et al. 2006a) as the distance of the Sun to the Galactic centre. Uncertainties in $d_{\odot}$ and $R_{\mathrm{GC}}$ are of the order of $0.1 \mathrm{kpc}$.
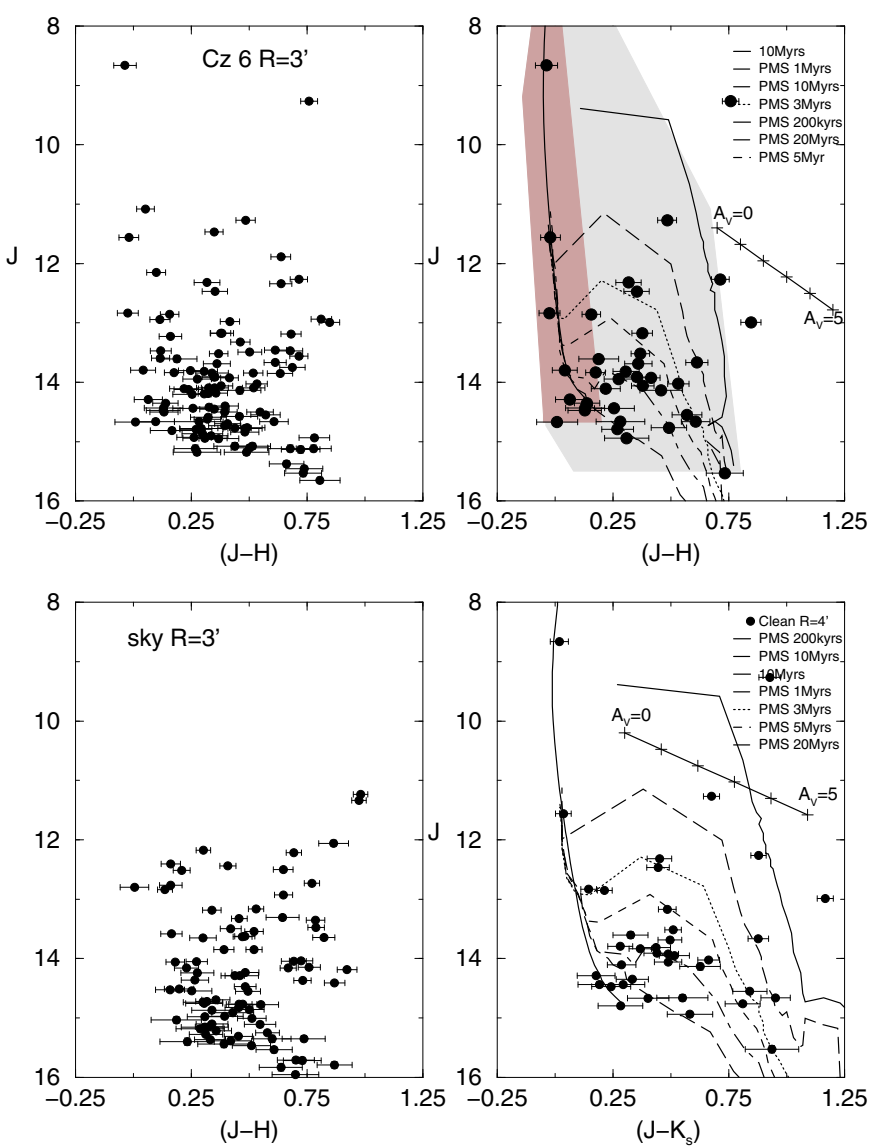

Fig. 8. Same as Fig. 7 for CMDs of the central regions $\left(R=4^{\prime}\right)$ of $\mathrm{Cz} 6$. Reddening vectors for $A_{V}=0-5$ are shown in decontaminated CMDs.

as the shaded region superimposed on the field-star decontaminated CMDs.

Colour-magnitude filters are only used to discard stars with colours comparable to those of the foreground/background field. This tool was previously applied to the structural analysis of the OCs M 67 (Bonatto \& Bica 2003), NGC 3680 (Bonatto et al. 2004b), NGC 188 (Bonatto et al. 2005), NGC 6611 (Bonatto et al. 2006b), NGC 4755 (Bonatto et al. 2006c), M 52 and NGC 3690 (Bonatto \& Bica 2006) and the faint OCs BH 63, Lyngå 2, Lyngå 12 and King 20 (Bica et al. 2006b). The filters were defined based on the distribution of the decontaminated star sequences in the CMDs of open clusters. They are wide enough to accommodate cluster MS and evolved star colour distributions, allowing for $1 \sigma$ photometric uncertainties. $\mathrm{CM}$ filter widths should also account for formation or dynamical evolution-related effects, such as enhanced fractions of binaries (and other multiple systems) towards the central parts of clusters, since such systems tend to widen the MS (e.g. Bonatto \& Bica 2007b; Bonatto et al. 2005; Hurley \& Tout 1998; Kerber et al. 2002). However, residual field stars with colours similar to those of the cluster are expected to remain inside the CM filter region. They affect the intrinsic RDP to a degree that depends on the relative densities of field and cluster stars. The contribution of the residual contamination to the observed RDP is statistically considered by means of the comparison field. In practical terms, the use of CM filters in cluster sequences enhances the contrast of the RDP against the background level, especially for objects in dense fields (see e.g. Sect. 4 in Bonatto \& Bica 2007b).

To avoid oversampling near the centre and undersampling for large radii, the RDPs were built by counting stars in 


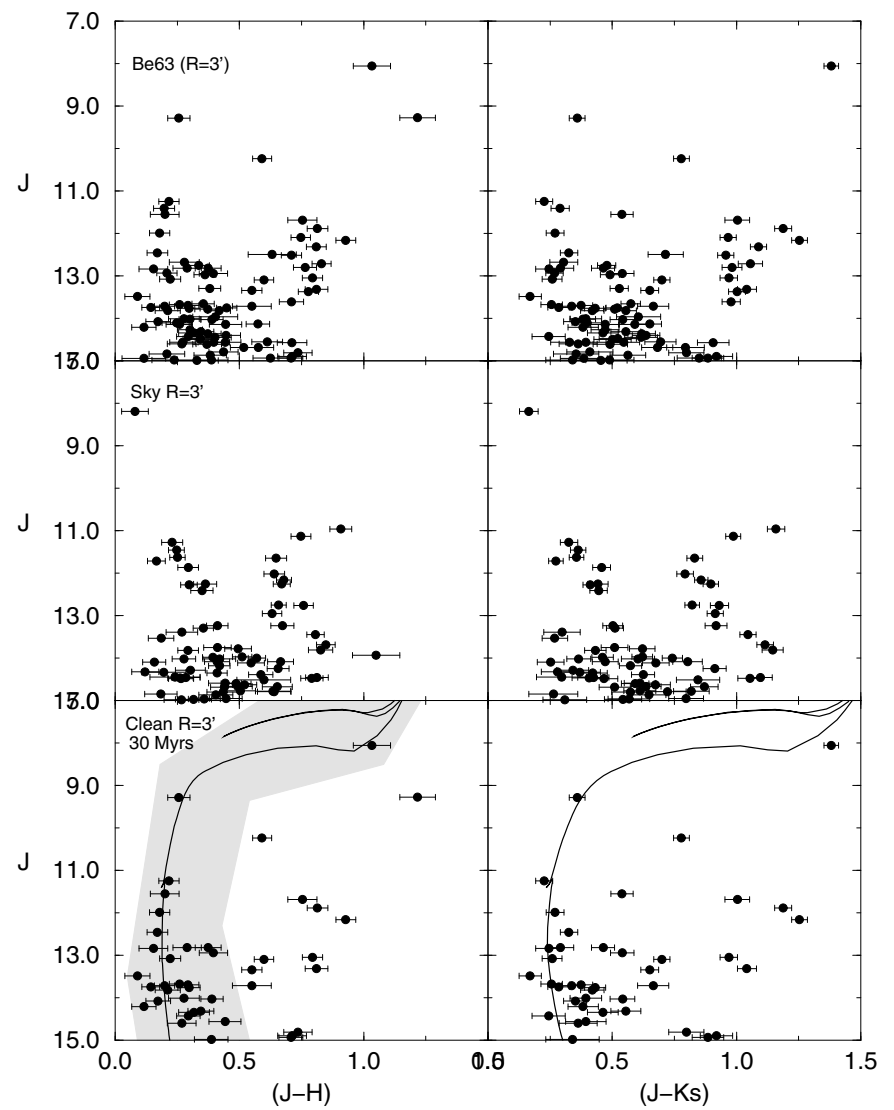

Fig. 9. 2MASS CMDs extracted from the $R=3^{\prime}$ region of Be 63. Top panels: observed CMDs $J \times(J-H)($ left $)$ and $J \times\left(J-K_{\mathrm{s}}\right)($ right $)$. Middle panels: equal area comparison field. Bottom panels: field star decontaminated CMDs fitted with the $30 \mathrm{Myr}$ Padova isochrone (solid line). The colour-magnitude filter used to isolate cluster MS/evolved stars is shown as a shaded region.

concentric rings of increasing width with distance to the centre. The number and width of rings are adjusted so that the resulting RDPs present adequate spatial resolution with moderate $1 \sigma$ Poisson errors. The $R$ coordinate (and respective uncertainty) of a given ring corresponds to the average distance to the cluster centre (and standard deviation) computed for the stars within the ring. The residual background level of each RDP corresponds to the average number of CM-filtered stars measured in the comparison field. Alternatively, we build RDPs with bins of variable sizes to check for any systematic biases that may have been introduced by our method. Following Maíz Apellániz \& Úbeda (2005) and Maschberger \& Kroupa (2009), we computed the RDPs with bins that contain a fixed number of stars, 10 for $0<R\left(^{\prime}\right)<1,100$ for $1<R\left(^{\prime}\right)<10$, and 1000 for $R>10^{\prime}$. Within the uncertainties, both approaches produce similar RDPs, as shown by the examples illustrated in Fig. 12.

Structural parameters were derived by fitting the twoparameter King (1966a) surface-density profile to the colour-magnitude filtered RDPs. The two-parameter King model essentially describes the intermediate and central regions of globular clusters (King 1966b; Trager et al. 1995). The fit was performed using a nonlinear least-squares fit routine that uses the errors as weights. The best-fit solutions are shown in Figs. 10 and 11 as a solid line superimposed on the RDPs. King's law is expressed as $\sigma(R)=\sigma_{\text {bg }}+\sigma_{0 K} /\left(1+\left(R / R_{\text {core }}\right)^{2}\right.$, where $\sigma_{\text {bg }}$ is the background surface density of stars, $\sigma_{0 K}$ is the central density of stars and $R_{\text {core }}$ is the core radius. The cluster radius $\left(R_{\mathrm{RDP}}\right)$

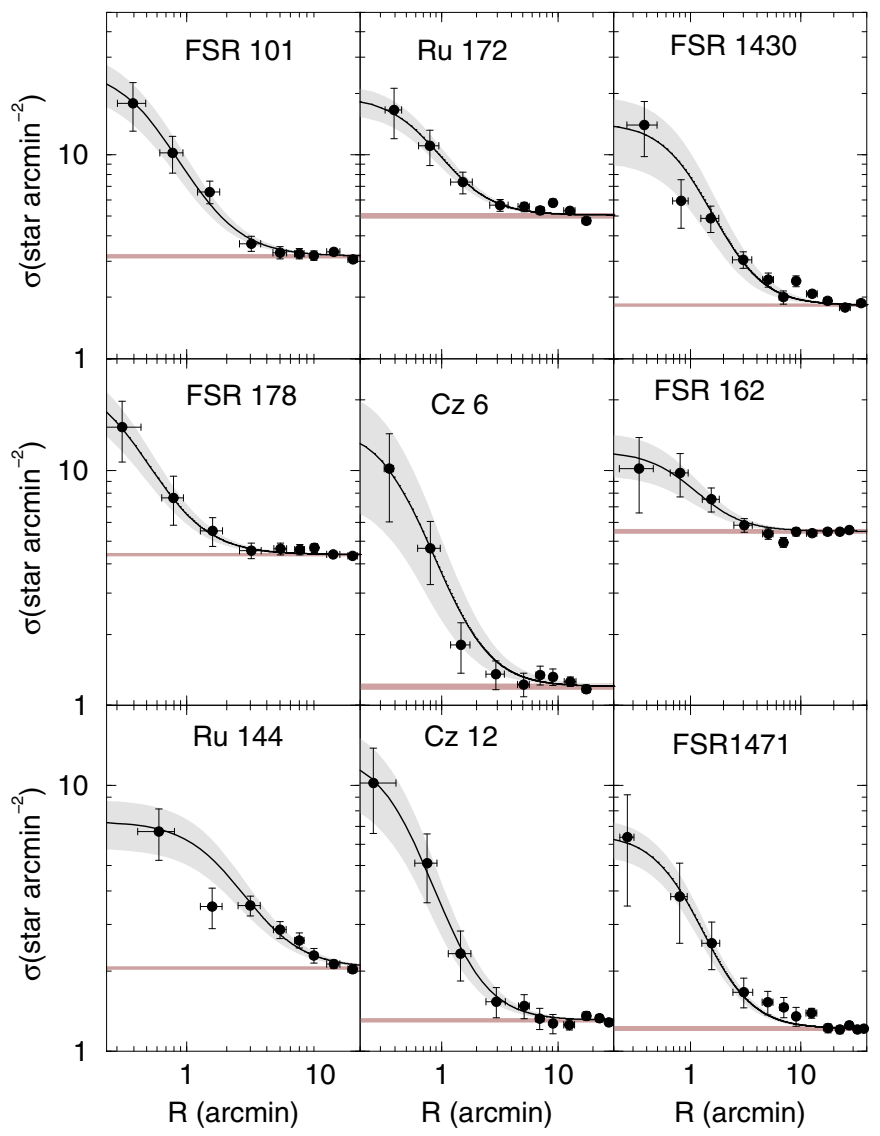

Fig. 10. Stellar RDPs (filled circles) built with colour-magnitude filtered photometry. Solid line: best-fit King profile. Horizontal shaded region: stellar background level measured in the comparison field. Gray regions: $1 \sigma$ King fit uncertainty.

and uncertainty can be estimated by considering the fluctuations of the RDPs with respect to the residual background, and $R_{\mathrm{RDP}}$ corresponds to the distance from the cluster centre where RDP and comparison field become statistically indistinguishable. The structural parameters derived are given in Table 4.

FSR 198 presents a conspicuous excess over the King-like profile in the innermost RDP bin (Fig. 11). Such a feature was been attributed to advanced dynamical evolution, having been detected in post-core collapse globular clusters (Trager et al. 1995). Some Gyr-old OCs, such as NGC 3960 (Bonatto \& Bica 2006) and LK 10 (Bonatto \& Bica 2009a), also present this cusp. However, very young OCs such as NGC 2244 (Bonatto \& Bica 2009b), NGC 6823 (Bica et al. 2008), Pismis 5, and NGC 1931 (Bonatto \& Bica 2009c) also display the RDP excess. Consequently, molecular cloud fragmentation and/or star formation effects probably play an important role in shaping the early stellar radial distribution of some OCs.

\section{Relations among astrophysical parameters}

At this point it is interesting to compare the structural parameters derived for the present OCs with those measured in different environments (Fig. 13). We considered (i) a sample of bright nearby OCs (Bonatto \& Bica 2005), including the two young OCs NGC 6611 (Bonatto et al. 2006b), and NGC 4755 (Bonatto et al. 2006c); (ii) OCs projected against the central parts of the Galaxy (Bonatto \& Bica 2007b); and (iii) the recently analysed OCs FSR 1744, FSR 89 and FSR 31 


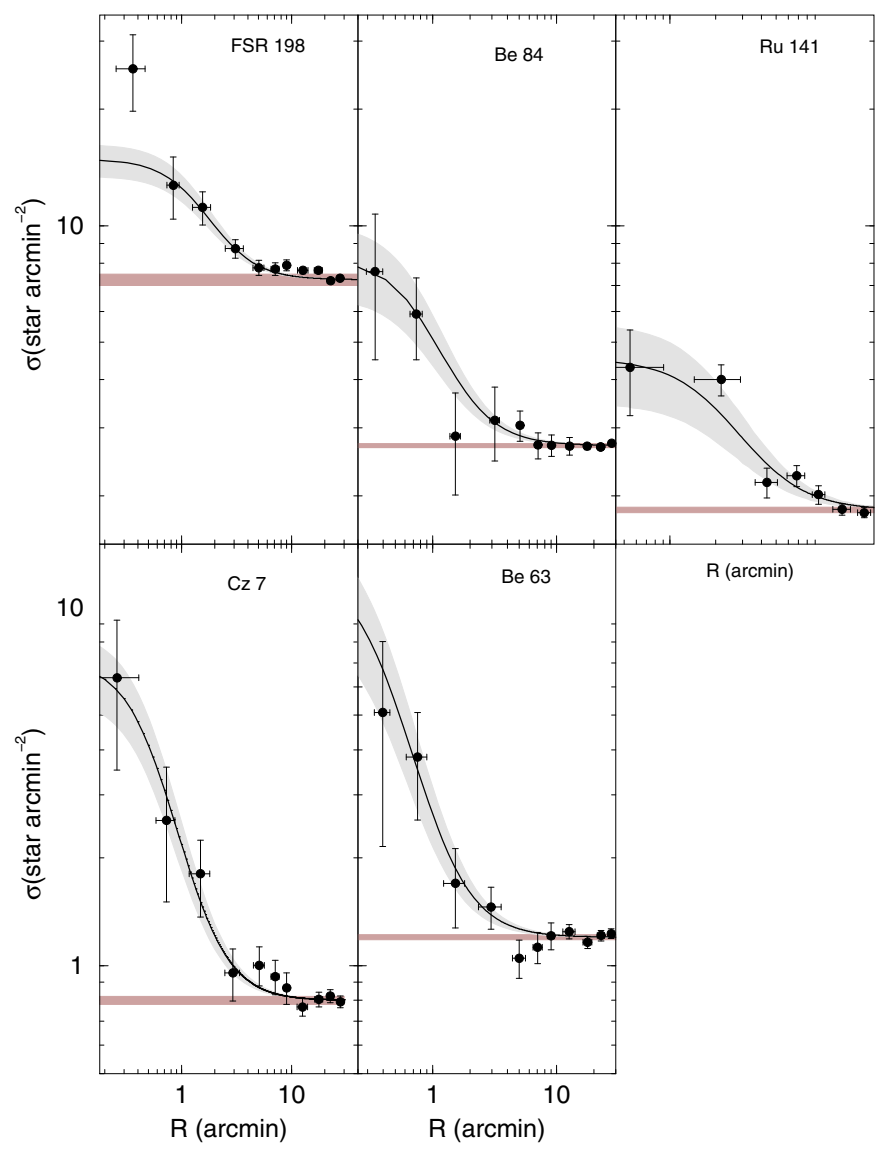

Fig. 11. Same as Fig. 10 for the remaining clusters.

(Bonatto \& Bica 2007a) projected against the central parts of the Galaxy; and (iv) the present sample.

The comparison OCs in the sample (i) have ages in the range $70 \mathrm{Myr}-7 \mathrm{Gyr}$, masses within 400-5300 $M_{\odot}$, and Galactocentric distances in the range $5.8 \lesssim R_{\mathrm{GC}}(\mathrm{kpc}) \lesssim 8.1$. NGC 6611 has $\approx 1.3 \mathrm{Myr}$ and $R_{\mathrm{GC}}=5.5 \mathrm{kpc}$, and NGC 4755 has $\approx 14 \mathrm{Myr}$ and $R_{\mathrm{GC}}=6.4 \mathrm{kpc}$. Sample (ii) OCs are characterised by $600 \mathrm{Myr} \lesssim$ age $\lesssim 1.3 \mathrm{Gyr}$ and $5.6 \lesssim R_{\mathrm{GC}}(\mathrm{kpc}) \lessgtr 6.3$. Sample (iii) consists of Gyr-class OCs at $4.0 \lesssim R_{\mathrm{GC}}(\mathrm{kpc}) \lesssim 5.6$.

In panel $(a)$ of Fig. 13, core and cluster radii of the OCs in sample (i) are almost linearly related by $R_{\mathrm{RDP}}=(8.9 \pm 0.3) \times$ $R_{\text {core }}^{(1.0 \pm 0.1)}$, which suggests that both kinds of radii undergo a similar scaling, in the sense that on average, larger clusters tend to have larger cores. However, $\frac{1}{3}$ of the OCs in sample (ii) do not follow that relation, which suggests that they are either intrinsically small or have been suffering important evaporation effects. The core and cluster radii in sample (iii) and the OCs of this work (iv) are consistent with the relation at the $1 \sigma$ level. A dependence of OC size on Galactocentric distance is shown in panel $(b)$, as previously suggested by Lyngå (1982) and Tadross et al. (2002). In panels $(c)$ and $(d)$ we compare core and cluster radii with cluster age, respectively. This relationship is intimately related to cluster survival/dissociation rates. Both kinds of radii present a similar dependence on age, in which part of the clusters expand with time, while some seem to shrink. The bifurcation occurs at an age $\approx 1$ Gyr. A similar effect was observed for the core radii of LMC and SMC star clusters (e.g. Mackey $\&$ Gilmore 2003$)$, which have core radii $\left(0.5 \lesssim R_{\mathrm{c}}(\mathrm{pc}) \lesssim 8\right)$ and mass $\left(10^{3} \lesssim M\left(M_{\odot}\right) \lesssim 10^{6}\right)$ significantly more than the present ones. The core radii distribution of most LMC and SMC clusters is characterised by a trend toward increasing core radius with

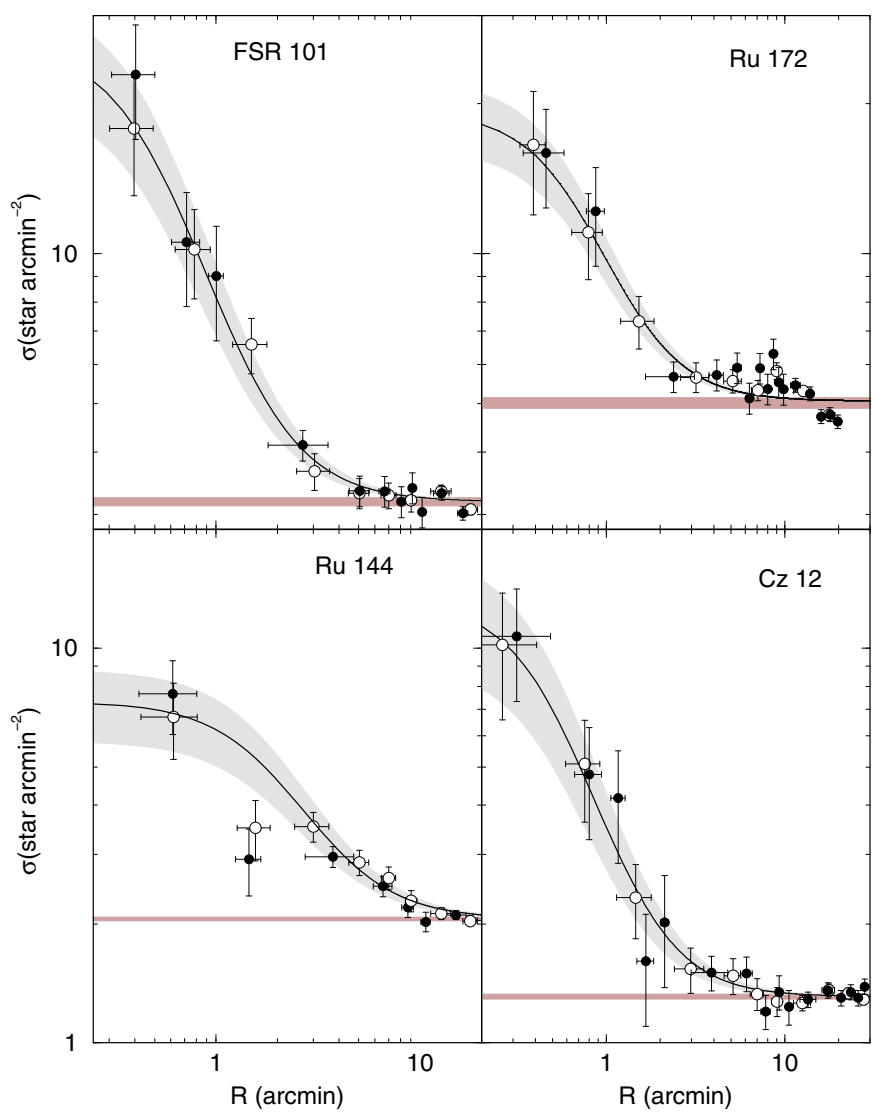

Fig. 12. Consistent RDPs are produced with our approach (empty circles) and that considering bins containing a fixed number of stars (filled).

age with an apparent bifurcation (core shrinkage) at several hundred Myr. Mackey \& Gilmore (2003) argue that this relationship represents true physical evolution, with some clusters developing expanded cores due to the stellar mass black-holes, and some that contract because of dynamical relaxation and core collapse (Mackey \& Gilmore 2008). We also note that the radii of the young clusters (age $<20 \mathrm{Myr}$ ) of our sample are related to the age similarly to the leaky ones of Pfalzner (2009). Similar relations involving core and cluster radii were found by Maciejewski \& Niedzielski (2007) for an optical cluster sample.

Finally, Fig. 14 shows the spatial distribution in the Galactic plane of the present OCs, compared to that of the OCs in the WEBDA database. We consider two age ranges, $<1$ Gyr and $>1$ Gyr. We compute the projections on the Galactic plane of the Galactic coordinates $(\ell, b)$. Old OCs are primarily found outside the solar circle, and the inner Galaxy contains the few OCs detected so far. The interesting point here is whether inner Galaxy clusters cannot be observed because of strong absorption and crowding, or have been systematically dissolved by the different tidal effects combined (Bonatto \& Bica 2007a, and references therein). In this context, the more OCs identified (with their astrophysical parameters derived) in the central parts, the more constraints can be established to settle this issue.

Differential reddening provides uncertainties in OC astrophysical parameters. Most OCs of our sample occur close to spiral arms. Since they are located close to the plane (Table 1), they may have interacted with the arms, especially by means of encounters with GMCs. 
Table 4. Structural parameters.

\begin{tabular}{|c|c|c|c|c|c|c|c|c|c|c|c|}
\hline Cluster & $\begin{array}{l}\left(1^{\prime}\right) \\
(\mathrm{pc})\end{array}$ & $\begin{array}{r}\sigma_{0 K} \\
\left(* \mathrm{pc}^{-2}\right)\end{array}$ & $\begin{array}{r}\sigma_{\mathrm{bg}} \\
\left(* \mathrm{pc}^{-2}\right)\end{array}$ & $\begin{array}{r}R_{\text {core }} \\
\text { (pc) }\end{array}$ & $\begin{array}{r}R_{\mathrm{RDP}} \\
(\mathrm{pc})\end{array}$ & $\begin{array}{r}\sigma_{0 K} \\
\left(*^{\prime-2}\right)\end{array}$ & $\begin{array}{r}\sigma_{\mathrm{bg}} \\
\left(*^{\prime-2}\right)\end{array}$ & $\begin{array}{r}R_{\text {core }} \\
\quad\left({ }^{\prime}\right)\end{array}$ & $\begin{array}{r}R_{\mathrm{RDP}} \\
\left.{ }^{\prime}{ }^{\prime}\right)\end{array}$ & $\begin{array}{r}\Delta R \\
\left({ }^{\prime}\right)\end{array}$ & $\mathrm{CC}$ \\
\hline (1) & (2) & (3) & (4) & (5) & (6) & (7) & (8) & (9) & (10) & (11) & (12) \\
\hline Be 63 & 1.64 & $4.2 \pm 1.5$ & $0.4 \pm 0.01$ & $0.6 \pm 0.1$ & $8.2 \pm 3.2$ & $11.5 \pm 4.0$ & $1.2 \pm 0.02$ & $0.38 \pm 0.08$ & $5.0 \pm 2.0$ & $15-30$ & 0.92 \\
\hline Be 84 & 0.50 & $21.9 \pm 6.9$ & $10.6 \pm 0.1$ & $0.4 \pm 0.1$ & $4.5 \pm 1.0$ & $5.5 \pm 1.7$ & $2.6 \pm 0.02$ & $0.2 \pm 0.1$ & $2.2 \pm 1.0$ & $15-30$ & 0.89 \\
\hline $\mathrm{Cz} 6$ & 0.81 & $21.8 \pm 12.0$ & $1.85 \pm 0.05$ & $0.37 \pm 0.2$ & $4.0 \pm 1.2$ & $14.2 \pm 7.8$ & $1.2 \pm 0.03$ & $0.46 \pm 0.2$ & $5.0 \pm 1.5$ & $10-20$ & 0.90 \\
\hline $\mathrm{Cz} 7$ & 0.95 & $6.8 \pm 1.6$ & $0.9 \pm 0.02$ & $0.5 \pm 0.08$ & $5.2 \pm 1.0$ & $6.28 \pm 1.5$ & $0.8 \pm 0.02$ & $0.53 \pm 0.09$ & $5.5 \pm 2.0$ & $10-30$ & 0.95 \\
\hline $\mathrm{Cz} 12$ & 0.57 & $35.8 \pm 12.6$ & $3.98 \pm 0.06$ & $0.27 \pm 0.08$ & $2.9 \pm 1.0$ & $11.8 \pm 4.13$ & $1.31 \pm 0.02$ & $0.5 \pm 0.2$ & $5.0 \pm 2.0$ & $10-20$ & 0.88 \\
\hline $\mathrm{Ru} 141$ & 0.53 & $2.6 \pm 1.4$ & $0.9 \pm 0.1$ & $1.6 \pm 0.6$ & $7.6 \pm 1.0$ & $0.7 \pm 0.4$ & $0.25 \pm 0.02$ & $3.0 \pm 1.2$ & $14.5 \pm 2.0$ & $10-20$ & 0.85 \\
\hline $\mathrm{Ru} 144$ & 0.47 & $23.8 \pm 6.8$ & $9.0 \pm 0.4$ & $0.9 \pm 0.2$ & $5.6 \pm 1.0$ & $5.3 \pm 1.5$ & $2.0 \pm 0.08$ & $1.95 \pm 0.36$ & $12.0 \pm 2.0$ & $12-20$ & 0.97 \\
\hline Ru 172 & 0.89 & $17.7 \pm 3.7$ & $6.3 \pm 0.06$ & $0.6 \pm 0.08$ & $6.1 \pm 1.0$ & $14.2 \pm 3.0$ & $6.3 \pm 0.06$ & $0.7 \pm 0.1$ & $6.8 \pm 1.0$ & $8-20$ & 0.96 \\
\hline FSR 101 & 0.54 & $79.7 \pm 21.3$ & $10.8 \pm 0.4$ & $0.28 \pm 0.04$ & $2.2 \pm 0.5$ & $23.5 \pm 6.3 \pm$ & $3.2 \pm 0.1$ & $0.52 \pm 0.09$ & $4.0 \pm 1.0$ & $8-20$ & 0.97 \\
\hline FSR 1 & 1.05 & \pm 5.0 & \pm 0.05 & 0.25 & $7.4 \pm 2.1$ & $13.0 \pm 5.4$ & $1.7 \pm 0.05$ & 0.24 & 2.0 & $20-40$ & 0.87 \\
\hline FSR & 0.78 & 4.95 & \pm 0.05 & $0.42 \pm 0.16$ & $5.5 \pm 1.6$ & $6.1 \pm 3.0$ & $0.97 \pm 0.03$ & 0.2 & $=2.0$ & $20-40$ & 0.80 \\
\hline FSR 162 & 2.05 & $5 \pm 0.6$ & \pm 0.01 & $1.88 \pm 0.6$ & $1.27 \pm 3.0$ & $6.54 \pm 2.5$ & $5.5 \pm 0.05$ & 0.3 & \pm 1.5 & $15-30$ & 0.88 \\
\hline FSR 178 & 1.07 & $9 \pm 5.0$ & $3.8 \pm 0.05$ & $0.36 \pm 0.06$ & $3.2 \pm 1.0$ & $20.6 \pm 5.7$ & $4.4 \pm 0.06$ & $0.34 \pm 0.06$ & $3.0 \pm 0.5$ & $10-20$ & 0.97 \\
\hline FSR 198 & 0.49 & $30.7 \pm 5.6$ & $29.2 \pm 0.2$ & $0.7 \pm 0.1$ & $3.43 \pm 1.0$ & $7.6 \pm 1.4$ & $7.2 \pm 0.06$ & $1.5 \pm 0.2$ & $7.0 \pm 2$ & $15-30$ & 0.98 \\
\hline
\end{tabular}

Notes. Column 2: arcmin to parsec scale. To minimise degrees of freedom in RDP fits with the King-like profile (see text), $\sigma_{\mathrm{bg}}$ was kept fixed (measured in the respective comparison fields) while $\sigma_{0}$ and $R_{\text {core }}$ were allowed to vary. Column 11: comparison field ring. Column 12: correlation coefficient.
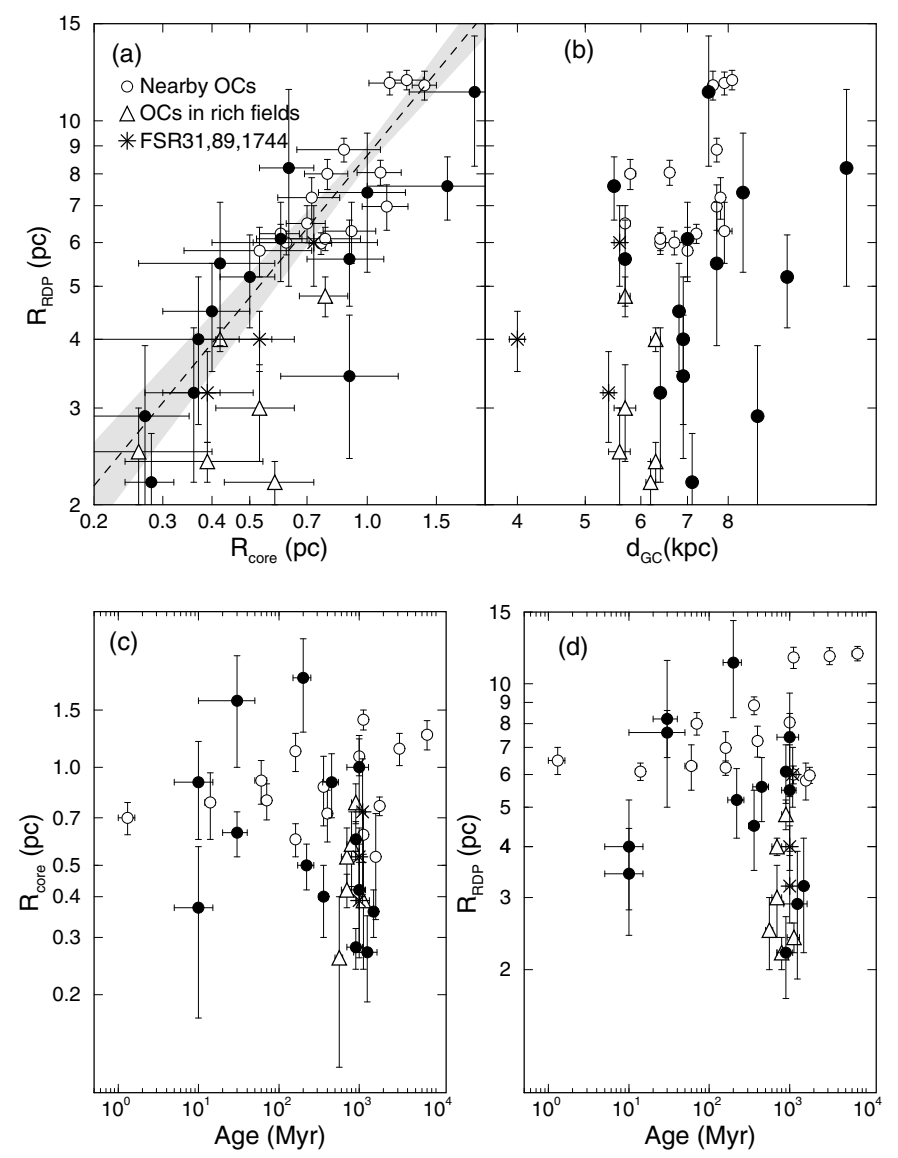

Fig. 13. Relations involving structural parameters of OCs. Empty circles: nearby OCs, including two young ones. Triangles: OCs projected on dense fields towards the centre. Stars: the similar OCs FSR 31, FSR 89 and FSR 1744. Black circles: the present work OCs.

\section{Concluding remarks}

In the present work, we have derived astrophysical parameters of 14 OCs projected close to the Galactic plane by means of 2MASS CMDs and stellar RDPs. Field-star decontamination is

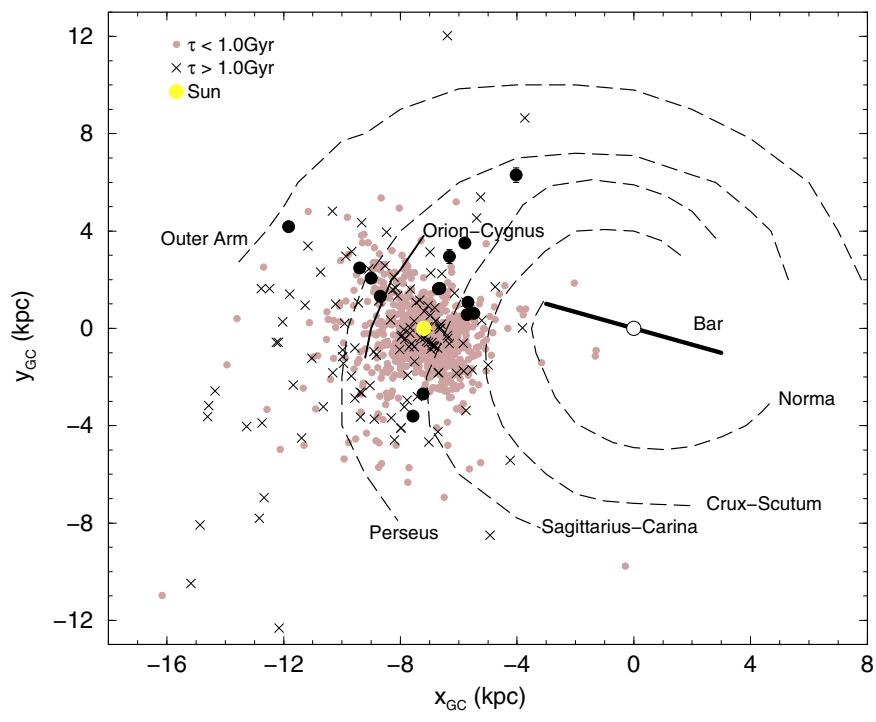

Fig. 14. Spatial distribution of the present star clusters (filled circles) compared to the WEBDA OCs with ages younger than 1 Gyr (gray circles) and older than 1 Gyr ("X"). The schematic projection of the Galaxy is seen from the North pole, with $7.2 \mathrm{kpc}$ as the Sun's distance to the Galactic centre.

applied to uncover the cluster's intrinsic CMD morphology, and CM filters are used to isolate probable cluster members. That field star decontamination leads to consistent CMDs and RDPs shows that we are dealing with OC, instead of field fluctuations. In particular, the present CMD and RDP analyses indicate that 6 IR objects from Froebrich et al. (2007), initially identified as cluster candidates from overdensities, are star clusters (Table 4).

Our sample contains OCs with ages in the range $10 \pm 5 \mathrm{Myr}$ (Cz 6 and FSR 198) to $1.5 \pm 0.5 \mathrm{Gyr}$ (FSR 178), at distances from the Sun in the range $d_{\odot} \approx 1.6 \mathrm{kpc}(\mathrm{Ru} 144)$ to $d_{\odot} \approx 7.1 \mathrm{kpc}$ (FSR 162) and Galactocentric distances $R_{\mathrm{GC}} \approx 5.5 \mathrm{kpc}$ for $\mathrm{Ru} 141$ to $R_{\mathrm{GC}} \approx 11.8 \mathrm{kpc}$ for $\mathrm{Be} 63$.

$\mathrm{Be} 84$, Ru 141 , and $\mathrm{Ru} 144$ are relatively young surviving OCs located inside the solar circle. Clusters in that region are expected to suffer important tidal stress in the form of shocks from disc and bulge crossings, as well as encounters with 
massive molecular clouds. In the long run, these processes tend to dynamically heat a star cluster, which enhances the rate of low-mass star evaporation and produces a cluster expansion on all scales. However, for some clusters, mass segregation and evaporation may also lead to a phase of core contraction. Consequently, these effects tend to disrupt most clusters, especially the less populous ones. On the other hand, FSR 1430, FSR 1471 and $\mathrm{Cz} 12$ are older. One of the reasons for such longevity may be their large Galactocentric distance, which minimises the disruption effects. The newly formed open clusters Cz 6 and FSR 198 show PMS stars in the CMDs.

The present study contributes new open cluster parameters and some revisions to the DAML02 and WEBDA open cluster databases.

Acknowledgements. We thank the anonymous referee for suggestions. We acknowledge support from the CNPq and CAPES (Brazil).

\section{References}

van den Bergh, S., \& McClure, R. D. 1980, A\&A, 88, 360 Bergond, G., Leon, S., \& Guilbert, J. 2001, A\&A, 377, 462 Bessel, M. S., \& Brett, J. M. 1988, PASP, 100, 1134

Bica, E., \& Bonatto, C. 2005, A\&A, 443, 465

Bica, E., Bonatto, C., Barbuy, B., \& Ortolani, S. 2006a, A\&A, 450, 105

Bica, E., Bonatto, C., \& Blumberg, R. 2006b, A\&A, 460, 83

Bica, E., Bonatto, C., \& Camargo, D. 2008, MNRAS, 385, 349

Bica, E., Bonatto, C., \& Dutra, C. 2008, A\&A, 489, 1129

Bonatto, C., \& Bica, E. 2003, A\&A, 405, 525

Bonatto, C., \& Bica, E. 2005, A\&A, 437, 483

Bonatto, C., \& Bica, E. 2006, A\&A, 455, 931

Bonatto, C., \& Bica, E. 2007a, A\&A, 473, 445

Bonatto, C., \& Bica, E. 2007b, MNRAS, 377, 1301

Bonatto, C., \& Bica, E. 2009a, MNRAS, 392, 483

Bonatto, C., \& Bica, E. 2009b, MNRAS, 394, 2127

Bonatto, C., \& Bica, E. 2009c, MNRAS, 397, 1915

Bonatto, C., Bica, E., \& Girardi, L. 2004a, A\&A, 415, 571

Bonatto, C., Bica, E., \& Pavani, D. B. 2004b, A\&A, 427, 485

Bonatto, C., Bica, E., \& Santos Jr., J. F. C. 2005, A\&A, 433, 917
Bonatto, C., Kerber, L. O., Santiago, B. X., \& Bica, E. 2006a, A\&A, 446, 121 Bonatto, C., Santos Jr., J. F. C., \& Bica, E. 2006b, A\&A, 445, 567

Bonatto, C., Bica, E., Ortolani, S., \& Barbuy, B. 2006c, A\&A, 453, 121

Dias, W. S., Alessi, B. S., Moitinho, A., \& Lepine, J. D. R. 2002, A\&A, 389, 871

Dutra, C. M., Santiago, B. X., \& Bica, E. 2002, A\&A, 383, 219

Friel, E. D. 1995, ARA\&A, 33, 38

Froebrich, D., Scholz, A., \& Raftery, C. L. 2007, MNRAS, 374, 399

Gieles, M., Portegies-Zwart, S., Athanassoula, E., et al. 2006, MNRAS, 371, 793

Gieles, M., Athanassoula, E., \& Portegies-Zwart, S. F. 2007, MNRAS, 376, 809

Girardi, L., Bertelli, G., Bressan, A., et al. 2002, A\&A, 391, 195

Hurley, J., \& Tout, A. A. 1998, MNRAS, 300, 977

Janes, K. A., \& Phelps, R. L. 1994, AJ, 108, 1773

Kerber, L. O., Santiago, B. X., Castro, R., \& Valls-Gabaud, D. 2002, A\&A, 390, 121

Kharchenko, N. V., Piskunov, A. E., Röser, S., Schilbach, E., \& Scholz, R.-D. 2005, A\&A, 438, 1163

King, I. 1966a, AJ, 71, 64

King, I. 1966b, AJ, 71, 276

Lamers, H. J. G. L. M., \& Gieles, M. 2006, A\&A, 455, 17

Lamers, H. J. G. L. M., Bastian, N., \& Gieles, M. 2004, A\&A, 429, 173

Lamers, H. J. G. L. M., Gieles, M., Bastian, N., et al. 2005, A\&A, 441, 117

Lyngå, G. 1982, A\&A, 109, 213

Maciejewski, G., \& Niedzielski, A. 2007, A\&A, 467, 1065

Mackey, A. D., \& Gilmore, G. F. 2003, MNRAS, 338, 120

Mackey, A. D., \& Gilmore, G. F. 2008, MNRAS, 386, 65

Maíz Apellániz, J., \& Úbeda, L. 2005, ApJ, 629, 873

Maschberger, T., \& Kroupa, P. 2009, MNRAS, 395, 931

Mermelliod, J. C. 1996, in The Origins, Evolution, and Destinies of Binary Stars in Clusters, ed. E. F. Milone, \& J. C. Mermilliod, ASP Conf. Ser., 90, 475

Oort, J. H. 1957, in Stellar populations, Rome, Pontifical Academy of Science, discussion in session on star clusters

Pavani, D. N., \& Bica, E. 2007, MNRAS, 468, 139

Pfalzner, S. 2009, A\&A, 498, 37

Piskunov, A. E., Kharchenko, N. V., Röser, S., Schilbach, E., \& Scholz, R.-D. 2006, A\&A, 445, 545

Siess, L., Dufour, E., \& Forestini, M. 2000, A\&A, 353, 593

Skrutskie, M. F., Cutri, R. M., Stiening, R., et al. 2006, AJ, 131, 1163

Tadross, A. L., Werner, P., Osman, A., \& Marie, M. 2002, NewAst, 7, 553

Tadross, A. L. 2008, MNRAS, 389, 285

Trager, S. C., King, I. R., \& Djorgovski, S. 1995, AJ, 109, 218

Wielen, R. 1971, A\&A, 13, 309

Wielen, R. 1988, The Harow Shapley Symposium on Globular Cluster Systems in Galaxies, ed. J. Grindlay, \& A. G. D. Philip (Dordrecht: Reidel), 393 